\title{
ELECTROMAGNETIC ANALYSIS USING TRANSMISSION LINE VARIABLES
}

\author{
Second Edition
}


This page intentionally left blank 


\section{ELECTROMAGNETIC ANALYSIS USING TRANSMISSION LINE VARIABLES Second Edition}

\section{Maurice Weiner}

Formerly with

U.S. Army Research Laboratory

and

United Silicon Carbide,Inc. 


\section{Published by}

World Scientific Publishing Co. Pte. Ltd.

5 Toh Tuck Link, Singapore 596224

USA office: 27 Warren Street, Suite 401-402, Hackensack, NJ 07601

UK office: 57 Shelton Street, Covent Garden, London WC2H 9HE

\section{British Library Cataloguing-in-Publication Data}

A catalogue record for this book is available from the British Library.

\section{ELECTROMAGNETIC ANALYSIS USING TRANSMISSION LINE VARIABLES (Second Edition)}

Copyright (c) 2010 by World Scientific Publishing Co. Pte. Ltd.

All rights reserved. This book, or parts thereof, may not be reproduced in any form or by any means, electronic or mechanical, including photocopying, recording or any information storage and retrieval system now known or to be invented, without written permission from the Publisher.

For photocopying of material in this volume, please pay a copying fee through the Copyright Clearance Center, Inc., 222 Rosewood Drive, Danvers, MA 01923, USA. In this case permission to photocopy is not required from the publisher.

ISBN-13 978-981-4287-48-7

ISBN-10 981-4287-48-2

Printed in Singapore. 


\section{To Carole, Tammy, Josh, Rebecca, and Steve}


This page intentionally left blank 


\section{Preface}

Over the past one hundred years or so, we have witnessed remarkable progress in expanding the frontiers of scientific knowledge. During this scientific journey, researchers have discerned many trends and themes. One of the most important of these has been the relentless preference of nature to discretize. Whether we look to the biological sciences and the double helix, or the quantization of electromagnetic energy, or the existence of quarks in particle physics, it appears nature loves to count, to compartmentalize, and to express all phenomena in terms of some sort of unit. With its virtually limitless capacity to control vast arrays of such individual elements, nature has endowed us with an amazing range of materials, life forms, and variegated phenomena.

Nature's affinity for using fundamental building blocks is not the whole story, however. Its capability in the realm of the infinitesimal is superseded by its incredible ability to synthesize, to derive function, and to obtain meaning from among these immense arrays of discretized elements, whether they be material units or bits of information. Nature indeed is the supreme "system engineer". An oft cited analogy is that drawn from certain impressionistic paintings. If looked at very closely, a limited portion of the painting appears as nothing more than a random collection of colored dots. Looked at from a distance, however, the painting takes on meaning and substance.

We therefore can think of nature as providing us with two viewpoints. One reflects a fundamental, building block perspective, and the other a cooperative phenomenon, which ties together the discrete elements, and in so doing gives rise to form, function, and meaning. These viewpoints are of course simplifications but nevertheless useful ones. The examples in nature are boundless: a nugget of coal consists of $10^{23}$ atoms or so, an organism consists of many cells, and so forth. By themselves these basic units are usually unrecognizable; but together these elements create new meaning. The designation of the building block is 
to some degree a matter of choice of course. The coal atoms consist of atomic as well as sub-atomic particles; the latter are not as useful in understanding the chemistry of coal and we therefore choose to ignore these units. On the other hand, we perceive atomic particles as having a decisive influence on the chemical properties.

Throughout the past century we have developed countless theories, many (but not all) of them containing fundamental units, or "building blocks", to help us understand natural phenomena. We have demanded of these theories self consistency, and of course the capability to explain known phenomena as well as to predict new phenomena. We have also insisted that they be consistent with other predictive theories. If the theory contained building blocks we have insisted that these units explain larger scale phenomena. In addition, although not absolutely necessary, we have attached extra value to theories which are mathematically "elegant".

The topic of classical electromagnetism, and the very important subset of electromagnetic wave propagation, certainly qualifies as an eminently "superb theory", to use the labeling of C. Penrose [1]. Despite the lack of any apparent building block unit (within the classical domain), the theory has exhibited unsurpassed predictive capabilities. In addition, the classical electromagnetic theory (essentially, Maxwell's Equations) has forged very successful links with other theories, such as quantum electrodynamics and relativity. We then raise the issue as to whether physical building blocks exist in the realm of wave propagation, including the classical regime? The use of the word "classical" would appear to contradict such an idea from the very outset. In this regard, we cannot point to any fundamental unit, or building block, except for its connections to quantum theory; in particular, the electromagnetic energy is quantized, with the elementary energy unit existing as a photon. In our discussion, however, we will forego the use of any units derived from quantum theory, assuming instead that the wavelength is relatively long and that the wave propagation remains in the classical regime.

Despite the lack of any apparent physical building block, we nevertheless proceed with the discretization of wave propagation phenomena. Indeed, the discretization process, applied to the electromagnetic propagation medium, forms the basis of this book. In view of the comments of the preceding paragraph, however, there are valid questions as to 
where and how the discretization process is to be introduced in the area of electromagnetics. In what follows we will imagine the propagation medium to be completely divided up into identical cells (for a given propagation velocity), with the electromagnetic energy confined to transmission lines, or "tracks", which separate the cells. Rather than relying on a fundamental building block derived from physics, the basic unit selected will be a mathematical one. The selection of the cell size is arbitrary, but we insist of course that as we reduce the size of the cell that the solution reduces to that of solving Maxwell's wave equation (including the effects of conductivity). We reiterate that the cell (and the cell size) is not an, irreducible, fundamental unit, but rather selected on the basis of mathematical convenience. The method is closely linked to numerical solutions of Maxwell's wave equation (the usual numerical approaches, however, do not take into account correlation effects described later).

In view of the previous comments, a question which immediately comes to mind is the following: is it worthwhile to re-introduce an old theory, dressed in new clothing, with an accompanying computer code for solving Maxwell's Equations? The answer is affirmative, and for three reasons. First, the transmission line method offers an extremely intuitive means for dealing with a wide assortment of electromagnetic propagation problems. Equilibrium, transient conductivity, and antenna problems are readily amenable to this method. From the outset a particular problem is viewed as a transmission line grid, rather than a purely numerical grid. Secondly the method provides an opportunity to discretize the propagation region, which can offer many new insights and may also be useful as a bridge to examine small scale effects when the cell size is allowed to shrink further in size, leaving behind the classical regime. Thirdly, the formulation allows one to study plane wave correlation effects in a convenient manner. Thus plane wave components in neighboring cells are aware of one another, leading to correlation effects which affects their plane wave properties. In this revised edition, the correlation/decorrelation theory has been refined and incorporated into the simulations

Hopefully this book will fill a niche not presently satisfied by two related types of books. The first type, stressing numerical methods for solving electromagnetic problems, lacks any appeal to physical intuition; very often the physics of electromagnetic propagation is lost because of 
the morass of mathematical detail, or the result of a physically unmotivated computer code. The second type of book, stressing transmission line techniques, has the desired physical appeal but is inadequate for solving two and three dimensional electromagnetic problems. This book bridges the gap between the two subject areas, illuminating the features common to both methods. Following the extensions and modifications to the transmission line theory, the model is applied to several illustrative electromagnetic problems. The book should have special appeal among electrical engineers and scientists involved with electromagnetic propagation and wideband transmitters/antennas. Although some background in transmission line theory is desirable, it is not essential since the needed background is provided.

What are some of the practical applications of the transmission line method? As alluded to in the previous paragraph, the method is particularly well suited for designing ultra-wideband or short pulse transmitters/antennas. The future will witness an inexorable drive to expand the bandwidth of transmitters/antennas, driven by the relentless needs of the information age. Engineers and scientists will need a more sophisticated understanding of picosecond devices, and its interaction with the environment, and will require computer codes capable of accurately describing such situations. The transmission line method should provide the engineer with the analytic and software tools necessary for dealing with this new technology. Besides laying the foundation for the transmission line method, the book also provides examples of computer codes which illustrate the transmission line technique. The code for the two dimensional solution of a photonic switch is provided is provided as an example.

The Chapters, outlined in the following, contain a fair amount of "new" material. We enclose new in quotes since the basic assumptions, embodied in Maxwell's Equations and elementary symmetry arguments, always represent the starting point. In most cases, however, the results are straightforward extensions of transmission line theory.

In this book we will deal almost exclusively with transmission lines situated on the borders of cubical cells (or squares, in the case of 2D), mainly as a matter of convenience. Neat geometrical cells, such as squares or cubes, are more amenable to mathematically analysis, and this is the primary reason for adopting such cells. Other cell geometries are possible, and in fact random irregularly shaped cells may be more ap- 
propriate, as we shall see. In any event the transmission line approach leads to an iterative solution of Maxwell's equations, which in turn may be translated into computer code.

Chapter 1 begins with a discussion of the many types of electromagnetic problems one can solve using transmission line matrix (TLM) techniques, whether they involve antennas, ultra-wideband sources, or even static potential problems. This is followed by a review of basic TLM theory, starting with the well documented, exact correspondence of the one dimensional wave equation and the transmission line solution. Once one ventures into two and three dimensions, however, the correspondence between the wave equation and the TLM matrix is not as apparent. Chapter 1 addresses these issues, pointing out the close relationship between standard numerical methods and the TLM matrix. Mathematically, we will see that the TLM method may be regarded as a particular type of finite difference method. Besides applications to electromagnetic propagation and other branches of applied physics, more flexible versions of the TLM matrix may be used to describe other diverse phenomena, for example, the modeling of neurological activity. In Chapter 2 the geometries and notation adapted for the TLM matrix are discussed. In many ways they are similar to the symmetry elements used to describe solid state crystals, in which the unit cells occupy the entire space. A systematic procedure for mapping the electromagnetic properties onto the TLM matrix, i.e., the transmission lines and the nodes, is discussed. Once the basic framework is established, Chapter 3 examines the electromagnetic scattering equations for one, two, and three dimensions. The scattering equations are very important, of course, since they are the hub of any computer iteration used to describe electromagnetic propagation. In Chapter 4 the TLM matrix is corrected for any plane wave properties which are usually present. The plane wave properties are studied by means of "correlations" between neighboring waves in adjacent transmission lines. The reverse process of "de-correlation", which removes plane wave behavior, also is introduced. This Chapter also deals with the inherent anisotropy present in the unit cells and the procedures needed to remove this effect. Plane wave and anisotropy effects are often ignored in the standard numerical methods. In this $2^{\text {nd }}$ Edition, the correlation/decorrelation procedures have been completely revised and many improvements introduced. Chapter 5 discusses the boundary conditions and dispersion. Dissimilar dielectrics, for example, 
will require different cell sizes, so the scattering at the interface becomes more complicated. The Chapter provides a systematic means for handling such boundary conditions. Embracing the effects of dispersion, as in Chapter 5, does not involve any fundamental obstacle, but its incorporation does make great demands on computer requirements. The TLM method is typically aimed at solving purely electromagnetic problems, namely, fast transient phenomena. However the same TLM framework also may be used to incorporate other phenomena, such as carrier drift, recombination, and charge separation. Chapter 6 addresses these issues. The main obstacle here is not so much the difficulty in incorporating such effects, but once again the substantial demands placed on computer memory and speed, brought about by combining fast and slow phenomena. In Chapter 7, an illustrative example is selected. We develop a computer program, based on the TLM method, for finding both the static (Laplace's Equation) and transient solutions of a 2D semicondutor switch, whose conductivity is induced by a light source. Unlike the First Edition, the computer simulations in the present edition incorporate plane wave correlations/decorrelations (PWC) into the computer code, which then generates both the static and transient solutions. The incorporation of PWC effects (as revised in Chapter 4) into the iteration allows for the accurate analysis of transient electromagnetic problems. Throughout the Chapter, it was found to be very useful to compare simulations with and without the inclusion of PWC effects. In addition, the actual program statements are provided, allowing readers to gain a better understanding of the method. Finally, in Chapter 8, we utilize existing software, such as SPICE. Such software may be applied to the TLM method when only a limited number of cells is required. Aside from the cell number limitations, other limitations inherent in SPICE involve the neglect of plane wave behavior, grid anisotropy, nonuniform propagation regions, boundary conditions, etc. Nevertheless, elementary but useful solutions may be obtained with SPICE, particularly one dimensional problems, and to a lesser extent, 2D problems. Several SPICE examples are discussed, which include RF transformers and pulse sources, as well as a simple description of a semiconductor switch.

The author's interest in transmission line methods, for solving electromagnetic problems, stems from his long and fruitful association with the Army Research Laboratory (ARL), Fort Monmouth, NJ. The Author has benefited from many discussions with scientists and engineers at 
ARL, and in particular, wishes to thank his many colleagues at the Pulse Power Laboratory for their interest and suggestions. The topics discussed in this book were initiated as a way of analyzing very fast conduction processes in semiconductor, for use in fast pulse generation and ultra- wideband sources. The author is presently with United Silicon Carbide, Inc. in New Jersey.

\section{REFERENCES}

1. Penrose, R. (1989), The Emperor's New Mind, Oxford University Press.

\section{NOTES ON SECOND EDITION}

The First Edition focused almost entirely on simulations which did not take into account plane wave effects. Although such simulation results are fine for static problems, the simulations are not capable of providing accurate answers for transient problems. To address this issue, this revised edition undertook two tasks. First the underlying theory (i.e., plane wave effects) was reworked and refined. Second, the associated iterations and simulations (based on the refined theory) was obtained, with the simulations incorporating plane wave effects from the outset.

The revised simulations are capable of solving complex transient, as well as static, electromagnetic problems using the same iteration. By incorporating plane wave effects the neighboring TLM cell waves are aware of one another, with the waves exhibiting correlation/ decorrelation behavior. The underlying theory for plane wave effects has been improved, making clearer the competition between the correlation and decorrelation mechanisms. The simulations produced in this manner are capable of greater accuracy in describing transient phenomena, when compared to other methods. As described in the revised book, the TLM method is capable of revealing subtle (and not so subtle) features of a transient electromagnetic signals-features which cannot be determined by other established methods (such as numerical methods) unless drastic revisions are undertaken.

In the revised book, the major revisions occur in Chapters 4 and 7. In Chapter 4 the underlying decorrelation model has been completely re- 
vised, which of course affects the way in which plane waves propagate. In Chapter 7, the simulations incorporate plane wave behavior from the outset (unlike the First Edition) which then are suitable for describing transient as well as static solutions. Since the simulations in the First Edition do not incorporate plane wave behavior, the resultant simulations cannot accurately describe the transient behavior. Nevertheless, as shown in Chapter 7, it is extremely useful to compare the two simulations (with and without plane waves). Suppose the final state solution is a static one. A requirement imposed on the plane wave simulations is that they arrive at the same final solution, regardless of whether the plane wave or the original (without plane wave correlations) simulations are used. The results of the comparison may then be used to improve the modeling of the correlation/decorrelation of the plane waves.

The revised book also takes into account semiconductors with arbitrary dielectric constant, using much smaller cell size, and thus extending the range of applicability and improving accuracy. These revisions are essential for accurately describing electromagnetic behavior under practical conditions.

Finally, in addition to the main revisions in Chapters 4 and 7, many important clarifications and corrections have been implemented in each of the Chapters. Redundant Figures and superfluous areas of discussion have been eliminated. Every effort has been made to correct or eliminate any unclear discussion. 


\section{CONTENTS}

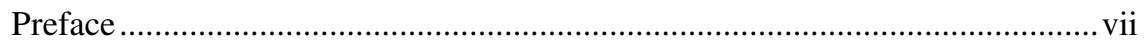

\section{INTRODUCTION TO TRANSMISSION LINES AND THEIR APPLICATION TO ELECTROMAGNETIC PHENOMENA ...1}

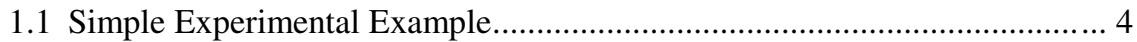

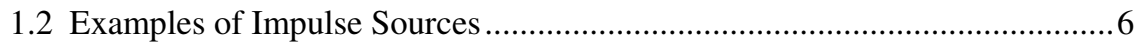

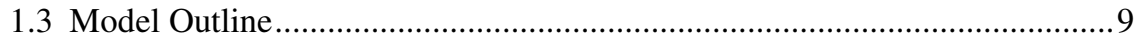

1.4 Application of Model to Small Node Resistance ….................................... 17

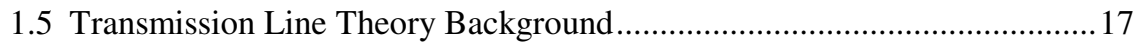

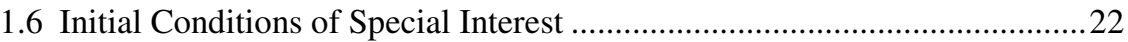

One Dimensional TLM Analysis. Comparison with Finite Difference

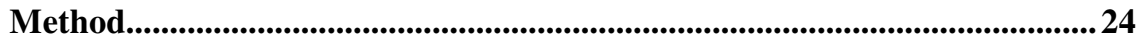

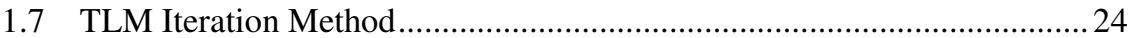

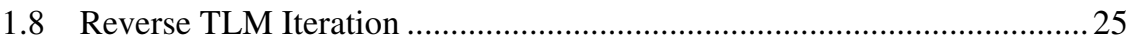

1.9 Derivation of Scattering Coefficients For Reverse Iteration......................29

1.10 Complete TLM Iteration (Combining Forward and Reverse Iterations)... 31

1.11 Finite Difference Method. Comparison with TLM Method........................32

Two Dimensional TLM Analysis. Comparison with Finite Difference

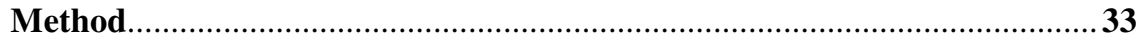

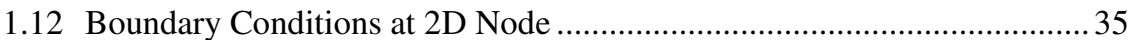

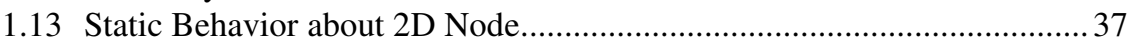

1.14 Non-static Example: Wave Incident on 2D node ........................................ 38

1.15 Integral Rotational Properties of Field about the Node ..............................42

1.16 2D TLM Iteration Method for Nine Cell Core Matrix ................................46

1.17 2D Finite Difference Method. Comparison with TLM Method .................50

1.18 Final Comments: Inclusion of Time Varying Signals and Phase

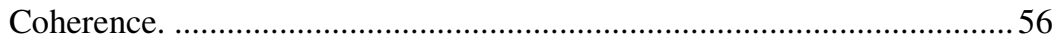

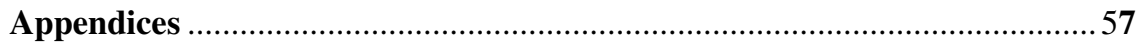

App. 1A.1 Effect of Additional Paths on Weighing Process ...............................57

App. 1A.2 Novel Applications of TLM Method: Description of Neurological Activity Using the TLM Method 
2.1 1D Cell Notation and Mapping of Conductivity and Field .........................67

2.2 Neighboring 1D Cells with Unequal Impedance ……..................................70

2.3 2D Cell Notation, Mapping of Conductivity and Field.................................72

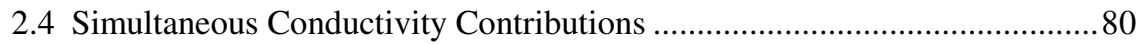

2.5 3D Cell Notation, Mapping of Conductivity and Field................................82

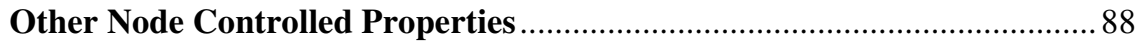

2.6 Node Control of 2D Scattering Coefficients Due to Finite Node

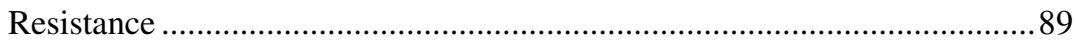

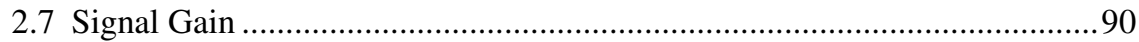

2.8 Signal Generation. Use of Node Coupling................................................ 91

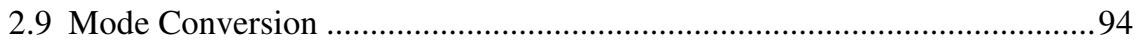

Example of Mapping: Node Resistance in Photoconductive

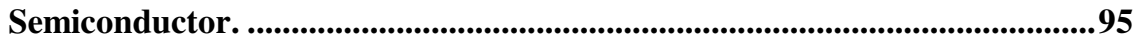

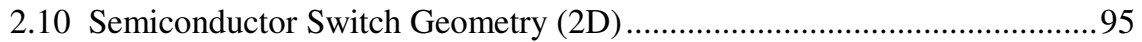

2.11 Node Resistance Profile in Semiconductor.............................................. 98

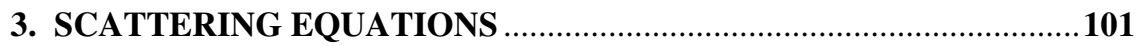

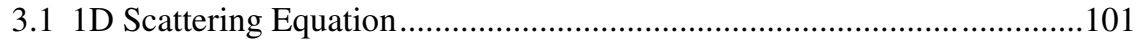

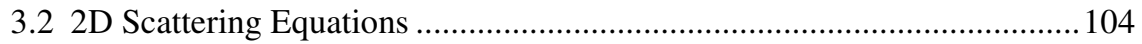

3.3 Effect of Symmetry on Scattering Coefficients........................................113

3.4 3D Scattering Equations: Coplanar Scattering..........................................116

General Scattering, Including Scattering Normal to Propagation

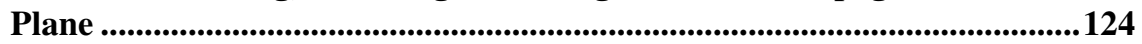

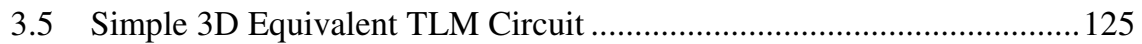

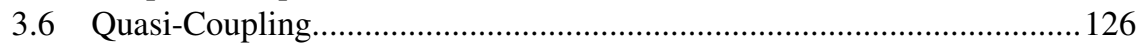

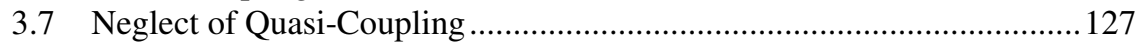

3.8 Simple Quasi-Coupling Circuit. First Order Approximation....................129

3.9 Correction to Quasi-Coupling Circuit: Second Order Approximation ...133

3.10 Calculation of Load Impedance with Quasi-Coupling ..............................136

3.11 Small Coupling Approximation of Second Order Quasi-Coupling ..........138

3.12 General 3D Scattering Process Using Cell Notation. .................................140

3.13 Complete Iterative Equations ………….............................................150

3.14 Contribution of Electric and Magnetic Fields to the Total Energy ...........153

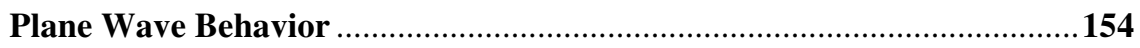

3.15 Response of 2D Cell Matrix to Input Plane Wave....................................154 
3.16 Response of 2D Cell Matrix to Input Waves with Arbitrary

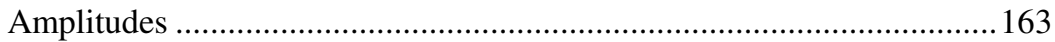

3.17 Response of 3D Cell Matrix to Input Plane Wave....................................164

3.18 Final Comments of Uniform Waves versus Plane Waves .........................167

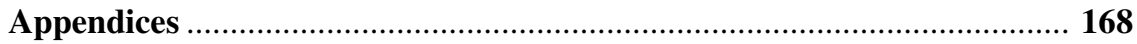

App.3A.1 Consistency of 3D Circuit with the TLM Static Solutions...............168

App. 3A.2 3D Scattering Coefficients, Without Quasi-Coupling in Terms of Circuit Parameters ...................................................................169

App. 3A.3 3D Scattering Coefficients with Both Coplanar and Aplanar Contributions into Unit Cell Lines (yz and zx Planes) .......172 App. 3A.4 3D Scattering Equations: with Both Coplanar and Aplanar Contributions into Unit Cell Lines (yz and zx Planes).

\section{CORRECTIONS FOR PLANE WAVES AND GRID ANISOTROPY EFFECTS}

4.1 Partition of TLM Waves into Component Waves

4.2 Scattering Corrections for 2D Plane Waves: Plane Wave Correlations Between Cells

4.3 Changes to 2D Scattering Coefficients 186

Corrections to Plane Wave Correlations

4.4 Correlation of Waves in Adjoining Media with Differing Dielectric Constants

4.5 Modification of Wave Correlation Adjacent a Conducting Boundary....... 190

Decorrelation Processes

4.6 Decorrelation Due to Sign Disparity of Plane and Symmetric Waves ..... 192

4.7 Related Scattering Criteria and Sign Conditions for Removal of the Sign Disparity .....

4.8 Minimal Solution Using Differing Decorrelation Factors to Remove Sign Disparities.

4.9 Decorrelation of Forward and Backward Plane Waves with Same Polarity in Neighboring Series TLM Lines Without Losses

4.10 Decorrelation of Forward and Backward Plane Waves with the Same

Polarity Occupying the Same TLM Line

4.11 Decorrelation Treatment at Boundary Interfaces

4.12 Comments on Interaction of Plane Wave Front with a Half-Infinite Conducting Plane

4.13 Summary of Correlation/Decorrelation Processes 
Treatment of Grid Orientation Effects

4.14 Dependence of Wave Energy Dispersal on Grid Orientation for

Symmetric and Plane Waves.............................................................2 213

4.15 Selection of Grid for Plane Waves ......................................................216

4.16 Transformation Properties between Grids ...........................................217

4.17 Possible Mini-Plane Wave Fronts Associated with Each Cell.

Plane Wave Partitioning .................................................................2 218

Grid(s) Selection. Propagation Vector Independence .............................220

4.18 Transformation of Fields to Principal Grid.......................................220

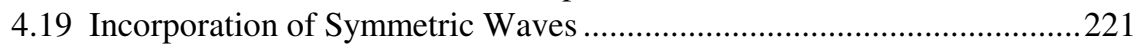

4.20 Iteration Method Using Principal Grid Transformations ....................... 222

4.21 Treatment of Separate TLM Correlated Wave Sources ..........................2224

4.22 Final Comments ...........................................................................226

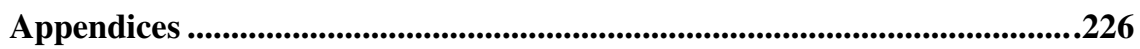

App. 4A.1 3D Scattering Corrections of Plane Waves (Plane Wave

Correlations) ...................................................................... 226

App. 4A.2 Consistency of Plane Wave Correlations with a Simple

Quantum Mechanical Model......................................................2229

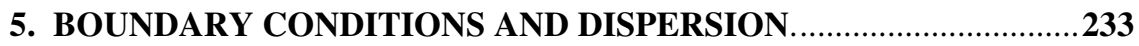

5.1 Dielectric-Dielectric Interface .................................................... 234

Node Coupling: Nearest Node and Multi-Coupled Node

Approximations …..............................................................................238

5.2 Nearest Nodes for 1D Interface ........................................................ 241

5.3 Nearest Nodes at 2D Interface..............................................................242

5.4 Truncated Cells and Oblique Interface ................................................... 244

5.5 Cell Index Notation at a Dielectric Interface Used in Simulations ............ 245

5.6 Simplified Iteration Neglecting the Nearest Node Approximation............247

5.7 Non-Uniform Dielectric. Use of Cluster Cells......................................248

Other Boundary Conditions ............................................................................251

5.8 Dielectric - Open Circuit Interface..................................................251

5.9 Dielectric - Conductor Interface....................................................2 252

5.10 Input/Output Conditions ............................................................... 254

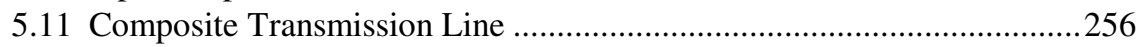

5.12 Determination of Initial Static Field by TLM Method..........................257 
Dispersion

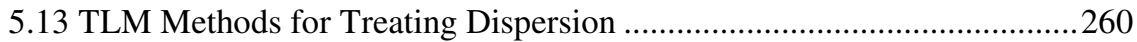

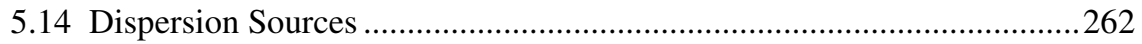

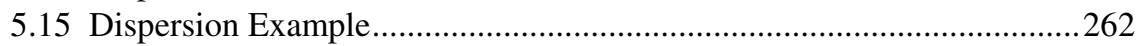

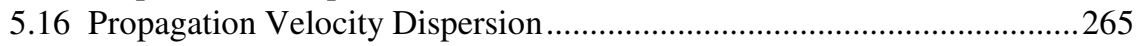

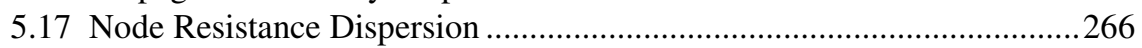

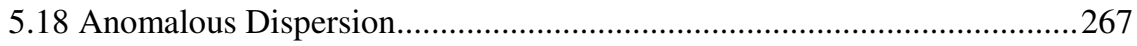

Incorporation of Dispersion into TLM Formulation..................................268

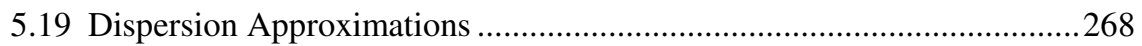

5.20 Outline of Dispersion Calculation Using the TLM Method......................269

5.21 One Dimensional Dispersion Iteration..................................................269

5.22 Initial Conditions with Dispersion Present ...........................................280

5.23 Stability of Initial Profiles with Dispersion Present.................................281

5.24 Replacement of Non-uniform Field in Cell with Effective Uniform

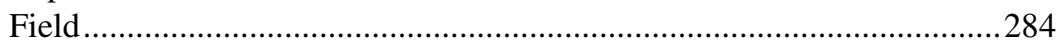

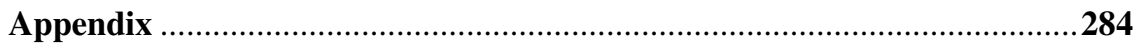

App. 5A.1 Specification of Input/Output Node Resistance to Eliminate

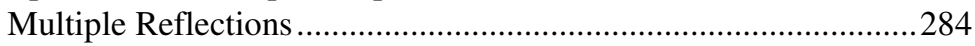

6. CELL DISCHARGE PROPERTIES AND INTEGRATION OF TRANSPORT PHENOMENA INTO THE TRANSMISSION

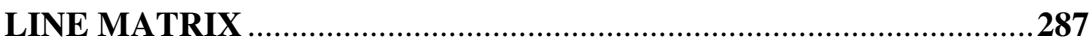

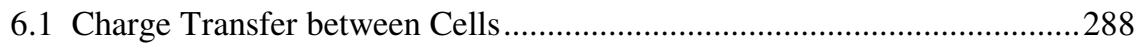

6.2 Relationship between Field and Cell Charge ............................................291

6.3 Dependence of Conductivity on Carrier Properties...................................295

Integration of Carrier Transport Using TLM Notation. Changes in Cell Occupancy and Its Effect on the TLM Iteration..................................296

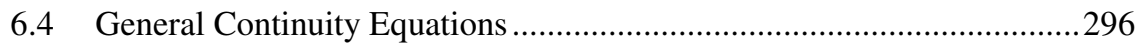

6.5 Carrier Generation Due to Light Activation..........................................296

6.6 Carrier Generation Due to Avalanching : Identical Hole and

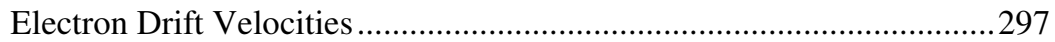

6.7 Avalanching with Differing Hole and Electron Drift Velocities ...............300

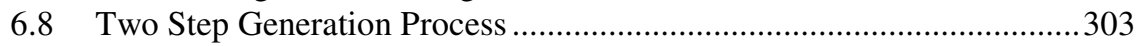

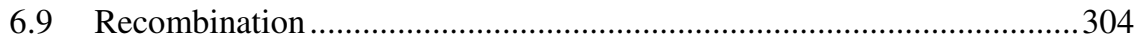

6.10 Limitations of Simple Exponential Recovery Model ...............................306

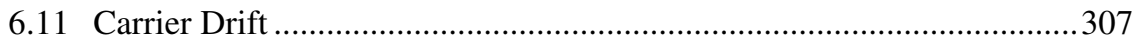


6.12 Cell Charge Iteration. Equivalence of Drift and Inter-Cell Currents

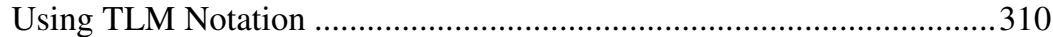

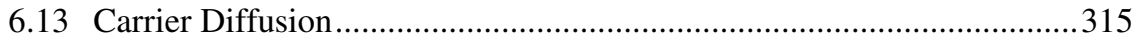

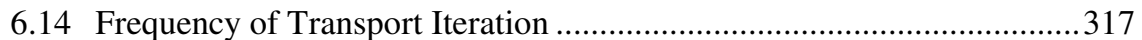

6.15 Total Contribution to Changes in Carrier Cell Occupancy ......................318

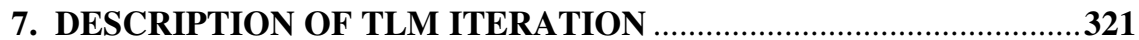

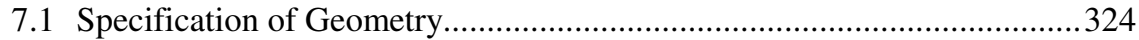

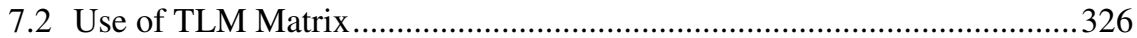

7.3 Various Regions which Incorporate Plane Wave Correlation/ Decorrelation (PWC Effects) into the Iteration

7.4 Simplified Decorrelation Procedure Used for Simulations in

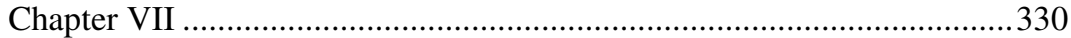

7.5 Description of Inputs, Arrays, and Initial Conditions.................................336

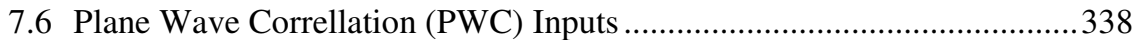

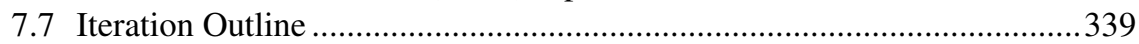

7.8 Node Resistance R(n,m) Changes. Use of Light Activation .....................340

Symmetric Scattering Simulations .............................................................................345

7.9 Symmetric Field Evolution with and without Node Activation ...................345

PWC Simulations. Comparison of PWC and Symmetric Results ................356

7.10 Comparison of Output Waveforms and Static Profiles for Symmetric

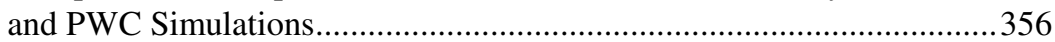

7.11 Comparison of Forward and Backward Waves when Using Wave Correlation. 362

7.12 Risetime and Alternating Field Effects in the Guided Region ..................364

7.13 Field Profile Evolution during Transient Charge-up Phase ......................366

7.14 Effect of Load Mismatch on Output and Field Profiles.............................368

7.15 Node Recovery and its Effect on Output Pulse and Field Profile.............370

7.16 Effects of Risetime on Conductivity.........................................................374

7.17 Partial Activation of Nodes and Effect on Profiles and Output ................375

7.18 Cell Charge Following Recovery ............................................................377

7.19 Role of TLM Waves at Charged Boundary ...........................................380

7.20 Incorporation of 3D Scattering Parameters into 2D Iteration. Application to Magnetostatic Solutions.....................................................381

7.21 Summary: Comparison of PWC and Symmetric Simulation Results.......383 Appendices .385

7A.1. Outline Discussion of Program Statements for Activated Semiconductor Switch 
7A.2 Program Statements for Optically Activated Semiconductor Switch.....397

7A.3 Matching Node Resistors for Input \& Output Sections .........................437

7A.4 Field Decay in Semiconductor Using the TLM Formulation..................438

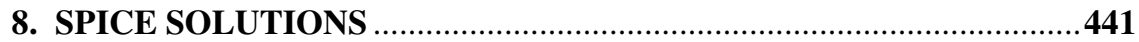

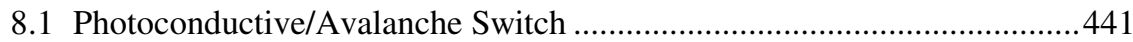

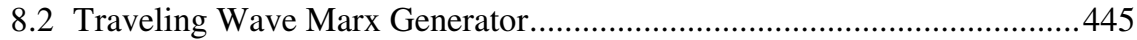

8.3 Traveling Marx Wave in a Layered Dielectric .........................................450

Pulse Transformation and Generation Using Non-Uniform

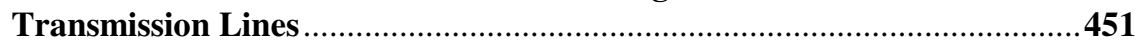

8.4 Use of Cell Chain to Simulate Pulse Transformer .....................................452

8.5 Pulse Transformer Simulation Results.....................................................455

8.6 Pulse Source Using Non-Uniform TLM Lines (Switch at Output)............456

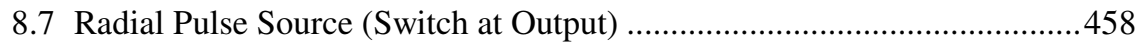

8.8 Pulse Sources with Gain (PFXL Sources) ……........................................459

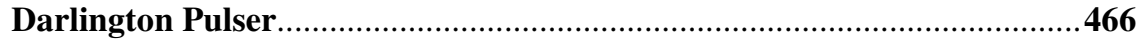

8.9 TLM Formulation of Darlington Pulser ...................................................469

8.10 SPICE Simulation of Lossy Darlington Pulser .......................................470

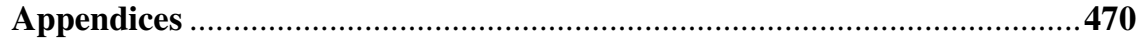

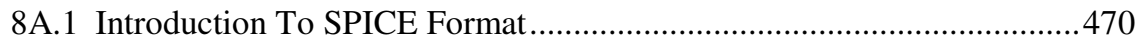

8A.2 Discussion of Format for Photoconductive Switch ..................................470

8A.3 TLM Analysis of Leading Edge Pulse in a Transformer..........................477

8A.4 TLM Analysis of Leading Edge Wave in PFXL.....................................480

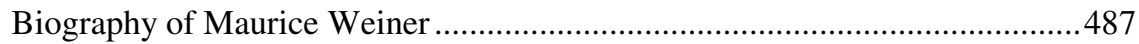

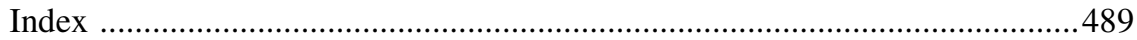

